\title{
Effects of Variations in Nonlinear Damping Coefficients on the Parametric Vibration of a Cantilever Beam with a Lumped Mass
}

\author{
Demian G. Silva ${ }^{1}$ and Paulo S. Varoto ${ }^{2}$ \\ ${ }^{1}$ Defense Projects, Empresa Brasileira de Aeronautica (EMBRAER), \\ Avenue Brigadeiro Faria Lima 2170, 12227-901 São José dos Campos, SP, Brazil \\ ${ }^{2}$ Mechanical Engineering Department, School of Engineering of Sao Carlos, \\ University of Sao Paulo, Avenue Trabalhador Saocarlense 400, 13566-590 São Carlos, SP, Brazil
}

Correspondence should be addressed to Paulo S. Varoto, varoto@sc.usp.br

Received 26 March 2008; Accepted 27 June 2008

Recommended by Jose Balthazar

Uncertainties in damping estimates can significantly affect the dynamic response of a given flexible structure. A common practice in linear structural dynamics is to consider a linear viscous damping model as the major energy dissipation mechanism. However, it is well known that different forms of energy dissipation can affect the structure's dynamic response. The major goal of this paper is to address the effects of the turbulent frictional damping force, also known as drag force on the dynamic behavior of a typical flexible structure composed of a slender cantilever beam carrying a lumped-mass on the tip. First, the system's analytical equation is obtained and solved by employing a perturbation technique. The solution process considers variations of the drag force coefficient and its effects on the system's response. Then, experimental results are presented to demonstrate the effects of the nonlinear quadratic damping due to the turbulent frictional force on the system's dynamic response. In particular, the effects of the quadratic damping on the frequency-response and amplitude-response curves are investigated. Numerically simulated as well as experimental results indicate that variations on the drag force coefficient significantly alter the dynamics of the structure under investigation.

Copyright ( 2008 D. G. Silva and P. S. Varoto. This is an open access article distributed under the Creative Commons Attribution License, which permits unrestricted use, distribution, and reproduction in any medium, provided the original work is properly cited.

\section{Introduction}

Characterization and quantification of uncertainties have been a topic of major importance in the context of structural dynamics. Generally speaking, the term uncertainty can be associated to variations of the system's physical parameters due to inaccuracies present either in the system's model or experimental data. In a broad view, the sources of uncertainties can be 


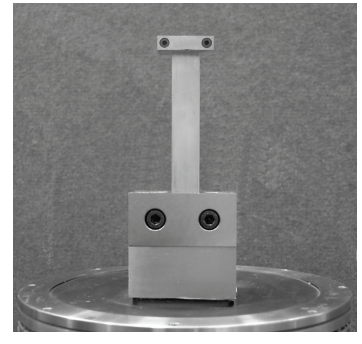

(a)

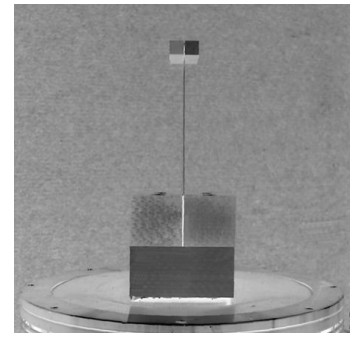

(b)

Figure 1: Physical system under investigation: (a) frontal view, (b) lateral view [19, 21, 23].

grouped into two main categories, namely statistical and nonstatistical [1], where the former is associated to fluctuations in the system's parameter mostly due to variations in material and/or geometry, and the later reflects inaccuracies present in the system's model caused by adoption of inappropriate assumptions or variations in numerical errors, for instance. More recently, a new terminology has been used to this classification by employing the words aleatory or aleatoric and epistemic $[2,3]$ to refer to these two groups of uncertainties, respectively. Several statistical and fuzzy theory-based procedures have been recently proposed (see [4-8]) to characterize and quantify uncertainties in complex structural systems. Similarly, uncertainties have also been subject of investigation in wave propagations and vibroacoustics (see [9-11]) as well as aerospace structures (see $[3,12,13]$ ). In the field of nonlinear structural dynamics, a reduced number of works have been reported. Nichols et al. [14] has developed a procedure for the detection of quadratic nonlinearities while Adhikari [2] has discussed uncertainties in damping models.

Parametrically excited cantilever beams have been extensively investigated in the last two decades, specially in the case of the principal parametric resonance [15-18]. Although most of these investigations have dealt with various aspects of the parametric resonance phenomenon, the majority of analysis was done by neglecting the effects of fluid medium. In this work, we perform an experimental and theoretical investigation on the effects of the viscous quadratic damping on the dynamic response of a cantilever beam with tip mass to a principal parametric resonance $[19,20]$. To investigate the quadratic damping effect, the structure shown in Figure 1 was built. It is composed of a slender stainless steel ASTM A240 beam, with dimensions of $100 \mathrm{~mm}$ in length, $20 \mathrm{~mm}$ in width, and $1 \mathrm{~mm}$ in thickness. The lumped mass is composed of carbon steel ASTM A36, with dimensions of $10 \mathrm{~mm}$ in length, $40 \mathrm{~mm}$ in width, and $20 \mathrm{~mm}$ in height. The opposite beam's end is clamped to a rigid base built from carbon ASTM A36 steel.

Figure 2 depicts the results of an experiment that was carried out by using the system shown in Figure 1. The experiment consisted of driving the structure into a principal parametric resonance condition through an input base sinusoidal signal. The structure's vibration and interaction with the surrounding fluid medium could be observed through the smoke-wire arrangement [19] as shown in the sequence of pictures of Figure 2. This experiment qualitatively indicates that the drag force plays an important role on the dynamics of the structure under investigation. Thus, this paper is concerned in studying theoretically and experimentally the effects of variations in the quadratic damping coefficients on the lateral vibration of a cantilever beam undergoing a principal parametric excitation. 


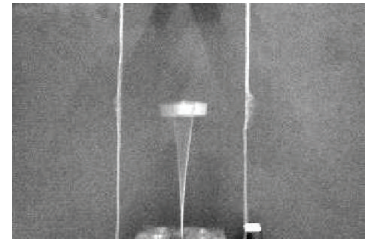

(a)

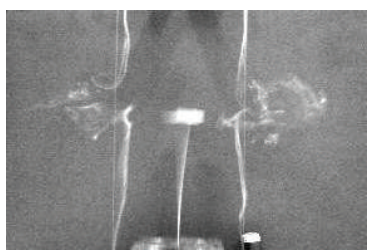

(d)

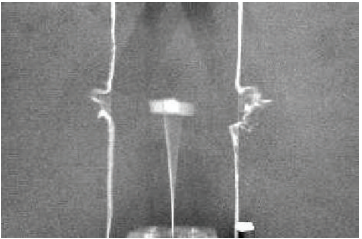

(b)

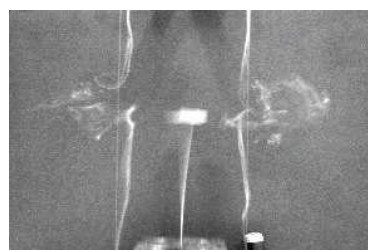

(e)

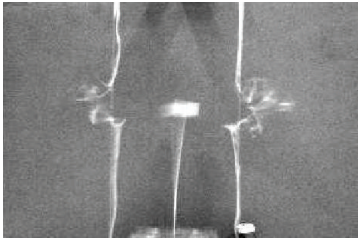

(c)

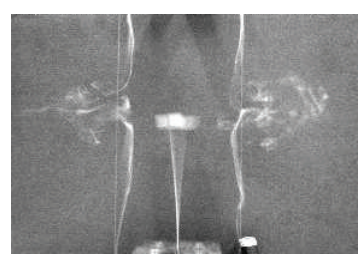

(f)

Figure 2: Qualitative effects of quadratic damping on the lateral vibration of the system [19].

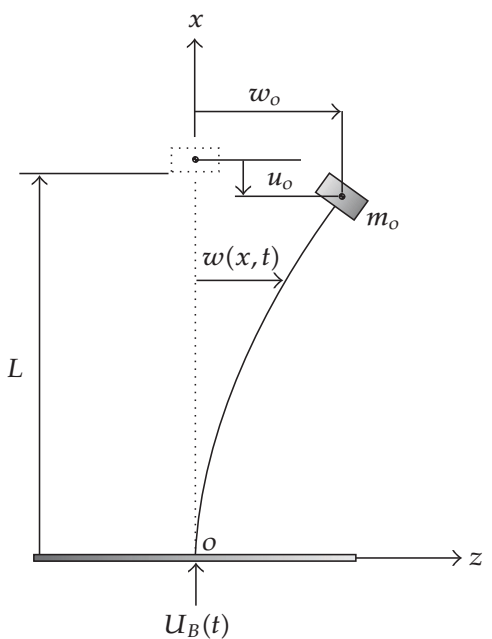

Figure 3: Physical model of the structure under investigation, combined acoustic, and base excitations [19].

\section{Mathematical modeling}

In a previous work by the authors [21], a general mathematical model for the structure shown in Figure 1 was developed by using the model shown in Figure 3. According to this model, the OXYZ orthogonal coordinates system is fixed at the base of the beam at its unstressed position and directed such that the $X$ axis is taken as the centerline of the beam. The origin $O$ of the coordinate system may be subject to a dynamic displacement $U_{B}(t)$ in the $X$ direction that represents the external driving signal. 


\subsection{Energy relationships}

The beam is modeled as a continuum solid with displacement field described by $u_{i}(i=1,2,3)$ and $u_{i}^{o}(i=1,2,3)$. The kinetic energy of the lumped-mass system can be described by

$$
T=\frac{1}{2} \int_{V} \rho \dot{u}_{i} \dot{u}_{i} d V+\frac{1}{2} m_{o} \dot{u}_{i}^{o} \dot{u}_{i}^{o}
$$

in which the dot denotes time derivative, $\rho$ and $V$ are, respectively, the material density and volume of the beam, and $m_{0}$ is the value of the lumped mass. In order to simplify (2.1), the contribution of the distributed mass of the beam will be ignored as well as the rotatory energy of the lumped mass. Hence, the kinetic energy is simplified to

$$
T=\frac{1}{2} m_{o}\left[\left(\dot{u}_{1}^{o}\right)^{2}+\left(\dot{u}_{3}^{o}\right)^{2}\right] \text {. }
$$

The task to find $T$ consists in performing several steps. First, the time derivative of the displacement field must be computed which results in expressions for $\left(\dot{u}_{1}\right)^{2}$ and $\left(\dot{u}_{3}\right)^{2}$. Second, the terms in the right-hand side of the expressions of $\left(\dot{u}_{1}\right)^{2}$ and $\left(\dot{u}_{3}\right)^{2}$ are described as functions of the $w(x, t)$ and its spatial derivatives. Third, a spatial reduction is necessary so that the deflection on the center of the lumped mass can be obtained. This can be done by using an expression of the form

$$
w(x, t)=\phi(x) w_{o}(t)
$$

in which $\phi(x)$ represents the first linear natural mode of the structure and $w_{o}(t)$ represents the modal coordinate associated with this natural mode. As a final result, the expressions for $\left(\dot{u}_{1}^{o}\right)^{2}$ and $\left(\dot{u}_{3}^{o}\right)^{2}$ are found and truncated to result in nonlinearities of third order as follows:

$$
\begin{gathered}
\left(\dot{u}_{1}^{o}\right)^{2}=\left(A_{1}\right)^{2} w_{o}^{2} \dot{w}_{o}^{2}-2 A_{1} w_{o} \dot{w}_{o} \dot{U}_{B}+\frac{8}{3} A_{2} w_{o}^{3} \dot{w}_{o} \dot{U}_{B}+\left(\dot{U}_{B}\right)^{2}, \\
\left(\dot{u}_{3}^{o}\right)^{2}=\dot{w}_{o}^{2} .
\end{gathered}
$$

Substituting the expressions of $\left(\dot{u}_{1}^{o}\right)^{2}$ and $\left(\dot{u}_{3}^{o}\right)^{2}$ described above into (2.2), the kinetic energy is then given as

$$
T=\frac{1}{2} m_{o} \dot{w}_{o}^{2}+\frac{1}{2} m_{o}\left[\left(A_{1}\right)^{2} w_{o}^{2} \dot{w}_{o}^{2}-2 A_{1} w_{o} \dot{w}_{o} \dot{U}_{B}+\frac{8}{3} A_{2} w_{o}^{3} \dot{w}_{o} \dot{U}_{B}+\left(\dot{U}_{B}\right)^{2}\right],
$$

in which $A_{1}$ and $A_{2}$ are geometrical constants given as

$$
\begin{aligned}
& A_{1}=\int_{0}^{L}\left(\frac{\partial \phi}{\partial x}\right)^{2} d x, \\
& A_{2}=\int_{0}^{L}\left(\frac{\partial \phi}{\partial x}\right)^{4} d x,
\end{aligned}
$$


and the first linear mode shape function $\phi(x)$ is given as

$$
\phi(x)=1-\cos \left(\frac{\pi x}{2 L}\right) .
$$

Once the final expression for the kinetic energy is known, the next step towards the derivation of equation of motion is obtaining the system's strain or potential energy which may be written as function of the stress and strain in the $X$ direction as

$$
U=\frac{1}{2} \iiint_{V} \sigma_{x x} \varepsilon_{x x} d V .
$$

By using the assumption that the material follows the constitutive Hooke law, and ignoring Poisson's effects, (2.8) reduces to

$$
U=\frac{1}{2} \iiint_{V} E \varepsilon_{x x}^{2} d V=\frac{1}{2} \int_{0}^{L} \int_{A} E \varepsilon_{x x}^{2} d A d x
$$

However, $u_{c}$ is very small when compared to $u_{r}$. Therefore, to simplify the analysis, the contributions of $u_{c}$ and of the gravitational field to the strain energy are ignored, thus giving

$$
U=\frac{1}{2} \int_{0}^{L} \int_{A} E\left[z^{2}\left(w^{\prime \prime}\right)^{2}-z^{2}\left(w^{\prime \prime}\right)^{2}\left(w^{\prime}\right)^{2}\right] d A d x .
$$

By writing $w(x, t)$ as a function of $\phi(x)$ and $w_{o}(t)$ (see (2.3)), the final expression for the strain energy truncated cubic terms in the system's equation of motion is given by

$$
U=\frac{1}{2} E I_{y} B_{1} w_{o}^{2}-\frac{3}{2} E I_{y} B_{2} w_{o}^{4}+\cdots,
$$

where $I_{y}$ is the area moment of inertial about the $Y$ axis, and the geometrical constants $B_{1}$ and $B_{2}$ are given as

$$
\begin{aligned}
& B_{1}=\int_{0}^{L}\left(\phi^{\prime \prime}\right)^{2} d x, \\
& B_{2}=\int_{0}^{L}\left(\phi^{\prime \prime}\right)^{2}\left(\phi^{\prime}\right)^{2} d x .
\end{aligned}
$$

The last step before deriving the system's equation of motion consists in obtaining the expression for the nonconservative forces acting on the system. Herein, it will be considered the action of two nonconservative forces. The first is the structural damping force which is modeled in terms of the generalized coordinates as $c_{1} \dot{w}_{0}$. The second is the aerodynamic drag damping force acting on the system (when in motion) and is proportional to the squared of the generalized velocity $c_{2} \dot{w}_{o}\left|\dot{w}_{o}\right|$. Both damping forces act in the negative direction of the virtual transversal displacement $\delta w_{0}$. Therefore, the nonconservative virtual work $\delta W_{\mathrm{nc}}$ which is done on the system is given by

$$
\delta W_{\mathrm{nc}}=\left(-c_{1} \dot{w}_{o}-c_{2} \dot{w}_{o}\left|\dot{w}_{o}\right|\right) \delta w_{o} .
$$

Since the nonconservative virtual work is defined as a function of the nonconservative generalized force $Q_{\mathrm{nc}}$ as $\delta W_{\mathrm{nc}}=Q_{\mathrm{nc}} \delta w_{\mathrm{o}}$, the generalized force $Q_{\mathrm{nc}}$ is obtained as

$$
Q_{\mathrm{nc}}=\frac{\delta W_{\mathrm{nc}}}{\delta w_{o}}=-c_{1} \dot{w}_{o}-c_{2} \dot{w}_{o}\left|\dot{w}_{o}\right| .
$$




\subsection{Equation of motion}

In the earlier section, the expressions for the kinetic energy $T$, strain energy $U$, and nonconservative generalized force $Q_{\mathrm{nc}}$ were obtained. From these results, it is possible to derive the system's equation of motion by using the well-known Lagrange equation [22] which, in turn, for the system under investigation is written as

$$
\frac{d}{d t}\left(\frac{\partial T}{\partial \dot{w}_{o}}\right)-\frac{\partial T}{\partial w_{o}}+\frac{\partial U}{\partial \dot{w}_{o}}=Q_{\mathrm{nc}}
$$

Through the computation of each term of Lagrange's equation and substitution of the result (2.15), the following result is obtained:

$$
\begin{gathered}
\left(1+\underline{A_{1}^{2} w_{o}^{2}}\right) \ddot{w}_{o}+\left(\frac{c_{1}}{m_{o}}+\frac{c_{2}}{\underline{m_{o}}\left|\dot{w}_{o}\right|}\right) \dot{w}_{o}+\left(\underline{A_{1}^{2} \dot{w}_{o}^{2}}-\underline{A_{1} \ddot{U}_{B}}+\frac{E I_{y} B_{1}}{m_{o}}\right) w_{o} \\
+\left[\frac{\underline{4} A_{2} \ddot{U}_{B}}{\left.\underline{\left(\frac{6 E I_{y} B_{2}}{m_{o}}\right)}\right]}\right] w_{o}^{3}=\frac{F(t)}{m_{o}}
\end{gathered}
$$

Equation (2.16) represents an ordinary inhomogeneous nonlinear time-dependent differential equation. In addition, this equation holds both the axial contraction and the curvature nonlinear effects. If both the underlined and double underlined terms are ignored, this equation reduces to a classical linear damped forced model. On the other hand, if only the double underlined terms are ignored, this equation reduces to the same equation obtained in [23] plus a forced term. Still, if the double underlined terms plus the nonlinear damping were ignored, this equation reduces to the same model obtained in [24] plus a forced term.

Since the present work is focused on the dynamic response of a structure under parametric sinusoidal excitation, it is considered that this excitation can be written as

$$
\ddot{U}_{B}(t)=-Q \lambda^{2} \cos (\lambda t+\varphi)=-Q_{o} \cos (\lambda t+\varphi)
$$

in which $Q_{o}$ is the magnitude of the input base acceleration, $\lambda$ is the parametric excitation frequency, and $\varphi$ is a phase shift. Then, (2.16) can be rewritten in the dimensional final form as

$$
\begin{aligned}
(1+ & \left.A_{1}^{2} w_{o}^{2}\right) \ddot{w}_{o}+\left(\frac{c_{1}}{m_{o}}+\frac{c_{2}}{m_{o}}\left|\dot{w}_{o}\right|\right) \dot{w}_{o}+\left(A_{1}^{2} \dot{w}_{o}^{2}+A_{1} Q_{o} \cos (\lambda t+\varphi)+\frac{E I_{y} B_{1}}{m_{o}}\right) w_{o} \\
& +\left[-\frac{4}{3} A_{2} Q_{o} \cos (\lambda t+\varphi)-\frac{6 E I_{y} B_{2}}{m_{o}}\right] w_{o}^{3}=0 .
\end{aligned}
$$

From the numerical viewpoint, it is interesting to work with the differential equation in dimensionless form. Therefore, by setting new dimensionless variables $w_{o}^{*}=A_{1} w_{o}$ and $t^{*}=t / T_{n}$ in which $T_{n}$ is the period of free vibration, the system's equation of motion is given by

$$
\left(1+w_{o}^{* 2}\right) \ddot{w}_{o}^{*}+H_{1} \dot{w}_{o}^{*}+H_{2} \dot{w}_{o}^{*}\left|\dot{w}_{o}^{*}\right|+\left[1+\dot{w}_{o}^{* 2}+H_{3} \cos \left(\Theta t^{*}+\varphi\right)\right] w_{o}^{*}-\left[H_{5}+H_{4} \cos \left(\Theta t^{*}+\varphi\right)\right] w_{o}^{* 3}=0
$$


in which

$$
\begin{gathered}
H_{1}=\frac{c_{1} T_{n}}{m_{o}}, \quad H_{2}=\frac{c_{2}}{m_{o} A_{1}}, \quad H_{3}=Q_{o} A_{1} T_{n}^{2}, \\
H_{4}=\frac{4 A_{2} Q_{o} T_{n}^{2}}{3 A_{1}^{2}}, \quad H_{5}=\frac{6 B_{2} E I_{y} T_{n}^{2}}{m_{o} A_{1}^{2}}, \\
\Theta=\lambda T_{n}, \quad \Psi=\Omega T_{n} .
\end{gathered}
$$

Further, we will discuss the effects of variations of the dimensionless quadratic damping coefficient $\mathrm{H}_{2}$ on the response of the parametrically driven cantilever beam.

\section{Perturbation analysis and numerical simulations}

In order to address the effects of the quadratic damping $\left(\mathrm{H}_{2}\right)$ on the structure's response, a solution of the (2.19) is required. Such a solution is here developed by employing the method of multiple scales (MME) [19]. For that purpose, it is more convenient to rewrite (2.19) in a slight different way by considering zero-order $\left(\varepsilon^{0}\right)$ and first-order $\left(\varepsilon^{1}\right)$ terms as follows:

$$
\begin{aligned}
\varepsilon^{0} \ddot{w}_{o}^{*}+\varepsilon^{0}(2 \pi)^{2} w_{o}^{*}= & -\varepsilon^{1} w_{o}^{* 2} \ddot{w}_{o}^{*}-\varepsilon^{1} \dot{w}_{o}^{* 2} w_{o}^{*}-\varepsilon^{1} H_{1} \dot{w}_{o}^{*}-\varepsilon^{1} H_{2} \dot{w}_{o}^{*}\left|\dot{w}_{o}^{*}\right| \\
& -\varepsilon^{1} H_{3} \cos \left(\Theta t^{*}+\varphi\right) w_{o}^{*}+\varepsilon^{1} H_{4} \cos \left(\Theta t^{*}+\varphi\right) w_{o}^{* 3}+\varepsilon^{1} H_{5} w_{o}^{* 3}
\end{aligned}
$$

in which the dimensionless coefficients $\varepsilon^{1} H_{1}, \varepsilon^{1} H_{2}, \ldots, \varepsilon^{1} H_{5}$ are defined according to (2.20). To apply the MME technique, first we express $w_{o}^{*}$ as

$$
w_{o}^{*}\left(\varepsilon ; t^{*}\right)=w_{0}\left(T_{0}, T_{1}\right)+\varepsilon w_{1}\left(T_{0}, T_{1}\right),
$$

where $T_{0}=t$ is the fast time scale associated with changes occurring at the frequency $\Theta$ and $T_{1}=\varepsilon^{1} t$ is a slow time scale associated with the modulations in amplitude and phase.

As it is known, the principal parametric resonance occurs when the parametric excitation $\Theta$ assumes a value that is equal to twice the undamped natural frequency $\omega_{n}$. Therefore, the normalized undamped natural frequency $\bar{\omega}_{n}=2 \pi$ can be written as

$$
(2 \pi)^{2}=\left(\frac{1}{2} \Theta\right)^{2}-\varepsilon \sigma
$$

in which $\varepsilon \sigma$ is a tuning parameter that flags the proximity of the principal parametric resonance.

By carrying out the standard details of the method of multiple scales, the first approximation to the solution of (3.1) is obtained as

$$
w_{o}^{*}=a\left(T_{1}\right) \cos \left[2 \pi T_{0}+\beta\left(T_{1}\right)\right]+O(\varepsilon)
$$

in which $a\left(T_{1}\right)$ and $\beta\left(T_{1}\right)$ are given by

$$
\begin{gathered}
a^{\prime}=\left(-\frac{1}{4 \Theta} \varepsilon H_{4} a^{3}+\frac{1}{2 \Theta} \varepsilon H_{3} a\right) \sin (2 \beta)-\frac{1}{2} \varepsilon H_{1} a-\frac{2}{3 \pi} \varepsilon H_{2} \Theta a^{2}, \\
a \beta^{\prime}=\left(-\frac{1}{2 \Theta} \varepsilon H_{4} a^{3}+\frac{1}{2 \Theta} \varepsilon H_{3} a\right) \cos (2 \beta)+\frac{\varepsilon}{8 \Theta}\left(-6 H_{5}-\Theta^{2}\right) a^{3}-\frac{\varepsilon \sigma a}{\Theta}
\end{gathered}
$$


and (3.5) are known as modulation equations. As stated in [19], steady state motions correspond to fixed points (constant solutions) of these modulation equations. Mathematically, this condition is reached when $a_{i}^{\prime}=\beta_{i}^{\prime}=0$. Hence, in steady-state condition the modulation equations are rewritten as

$$
\begin{gathered}
\left(-\frac{1}{4 \Theta} \varepsilon H_{4} \bar{a}^{3}+\frac{1}{2 \Theta} \varepsilon H_{3} \bar{a}\right) \sin (2 \beta)-\frac{1}{2} \varepsilon H_{1} \bar{a}-\frac{2}{3 \pi} \varepsilon H_{2} \Theta \bar{a}^{2}=0, \\
\left(-\frac{1}{2 \Theta} \varepsilon H_{4} \bar{a}^{3}+\frac{1}{2 \Theta} \varepsilon H_{3} \bar{a}\right) \cos (2 \beta)+\frac{1}{8 \Theta}\left(-6 \varepsilon H_{5}-\Theta^{2}\right) \bar{a}^{3}-\frac{\varepsilon \sigma \bar{a}}{\Theta}=0,
\end{gathered}
$$

where $\bar{a}$ describes the steady-state vibration amplitude. Trivial solutions of this system of equations are immediately apparent and correspond to the case where $\bar{a}=0$. Nontrivial solutions $(\bar{a} \neq 0)$ are obtained by solving (3.6) in terms of the amplitude $\bar{a}$ and phase angle $\beta$. The solution process for these quantities is laborious and can be found in detail in the work by da Silva [19]. The final expression for $\bar{a}$ is given as

$$
\bar{a}= \pm \frac{\sqrt{-2 E_{4} E_{2} \pm 2 E_{4} \sqrt{E_{2}^{2}-4 E_{4} E_{0}}}}{2 E_{4}}
$$

where the $E_{i}$ are given as

$$
\begin{aligned}
E_{4}= & -9 \pi^{2} \varepsilon H_{4} \varepsilon H_{3} \Theta^{2} \varepsilon \sigma-54 \pi^{2} \varepsilon H_{4} \varepsilon H_{3} \varepsilon H_{5} \varepsilon \sigma+9 \pi^{2} \varepsilon H_{4}^{2} \varepsilon \sigma^{2}-\frac{117}{4} \pi^{2} \varepsilon H_{4}^{2} \varepsilon H_{3}^{2} \\
& -32 \Theta^{4} \varepsilon H_{4} \varepsilon H_{3} \varepsilon H_{2}^{2}+\frac{9}{16} \pi^{2} \varepsilon H_{3}^{2} \Theta^{4}+\frac{81}{4} \pi^{2} \varepsilon H_{3}^{2} \varepsilon H_{5}^{2}+\frac{27}{4} \pi^{2} \varepsilon H_{3}^{2} \varepsilon H_{5} \Theta^{2}, \\
E_{2}= & 27 \pi^{2} \varepsilon H_{4} \varepsilon H_{3}^{3}+54 \pi^{2} \varepsilon H_{3}^{2} \varepsilon H_{5} \varepsilon \sigma+16 \Theta^{4} \varepsilon H_{3}^{2} \varepsilon H_{2}^{2}+9 \pi^{2} \varepsilon H_{3}^{2} \Theta^{2} \varepsilon \sigma-36 \pi^{2} \varepsilon H_{4} \varepsilon H_{3} \varepsilon \sigma^{2}, \\
E_{0}= & 36 \pi^{2} \varepsilon H_{3}^{2} \varepsilon \sigma^{2}-9 \pi^{2} \varepsilon H_{3}^{4},
\end{aligned}
$$

and the phase angle $\beta$ is written as

$$
\beta=\frac{1}{2} \tan ^{-1}\left(\frac{G_{1}}{G_{2}}\right)
$$

with coefficients $G_{1}$ and $G_{2}$ given as

$$
\begin{aligned}
G_{1}= & 8 \Theta\left(4 \varepsilon H_{2} \Theta \varepsilon H_{4} \bar{a}^{3}-4 \varepsilon H_{2} \Theta \varepsilon H_{3} \bar{a}\right), \\
G_{2}= & 3 \pi\left(6 \varepsilon H_{5} \varepsilon H_{4} \bar{a}^{4}+\varepsilon H_{4} \Theta^{2} \bar{a}^{4}+8 \varepsilon H_{4} \varepsilon \sigma \bar{a}^{2}\right) \\
& +3 \pi\left(-12 \varepsilon H_{3} \varepsilon H_{5} \bar{a}^{2}-2 \varepsilon H_{3} \Theta^{2} \bar{a}^{2}-16 \varepsilon H_{3} \varepsilon \sigma\right) .
\end{aligned}
$$

Hence, the solution for the modulation equations is given as a function of the parametric excitation frequency $\Theta$, the frequency tuning parameter $\varepsilon \sigma$, and the dimensionless coefficients $H_{i}$. Particularly, we are interested in investigating the effects of variations of the dimensionless quadratic damping coefficient $\mathrm{H}_{2}$ on the amplitude of the response. For that purpose, a series of simulations were performed by varying this parameter on the above equations and computing 
the resulting response. Figure 4 shows the computed response $\bar{a}$ from (3.7) for different values of $\varepsilon H_{2}$. It is seen that variations of this parameter do not alter the critical points $C$ and $E$. Additionally the nonsymmetric shape of the amplitude-frequency curve tends to decrease as $\varepsilon \mathrm{H}_{2}$ increases. It is also noticed that two characteristics of the response are strongly influenced by variations on the quadratic damping coefficient, namely, the amplitude of the response and stability of the nontrivial ramification CG. The maximum value of the amplitude of response $\bar{a}_{\max }$ represents an important information of the system under investigation. In this sense, it is equally important to assess the influence of variations of the nonlinear damping coefficient on $\bar{a}_{\text {max }}$. Figure 5 shows the behavior of this parameter when variations on $\varepsilon H_{2}$ are introduced. The results were obtained for three different values of the amplitude of the parametric excitation, represented by the dimensionless coefficient $\varepsilon H_{3}$. Figure 5 (a) shows that for small values of $\varepsilon H_{2}\left(0<\varepsilon H_{2}<0.1\right), \bar{a}_{\max }$ is relatively insensitive to variations on $\varepsilon H_{2}$. The largest impact of $\varepsilon H_{2}$ on $\bar{a}_{\max }$ occurs in the $0.2<\varepsilon H_{2}<0.5$ range, becoming less sensitive as $\varepsilon H_{2}$ approaches the end of the range. Figure 5(b) shows essentially the same trend where the values of the relative reduction of the amplitude are depicted. As previously pointed out in Figure 4, the stability of the nontrivial ramification CG is strongly affected by the nonlinear quadratic damping since it involves the definition of the bifurcation shown in point $G$. This bifurcation is responsible for the jump phenomenon when the values of the tuning parameter $\varepsilon \sigma$ are varied in the ascending order. From the numerical solution of (3.7) we can identify critical values of $\varepsilon H_{2}$ in the response-frequency curve that will make the bifurcation disappear. These critical values can be found from the following expression:

$$
\varepsilon H_{2}^{\text {crit }}=-0.012 \varepsilon H_{3}^{2}+0.14 \varepsilon H_{3}+0.14
$$

and they are represented in Figure 6. Hence, from this equation it is possible to estimate values for the $\varepsilon H_{2}$ for a given known excitation condition $\left(\varepsilon H_{3}\right)$ that would make the jump phenomenon to completely disappear from the system's response. Figure 7 shows the effects of variations of the $\varepsilon H_{2}$ in the system's response when the excitation amplitude is varied. It can be seen that the critical point $\mathrm{C}$ is not affected by the different values of $\varepsilon \mathrm{H}_{2}$. On the other hand, the vibration amplitude and the stability of the nontrivial ramification $C F$ are strongly affected when varying the values of the quadratic damping coefficient. It is also interesting to observe in Figure 7 the magnitude of the response at $D$ and $F$, here referred to as $\bar{a}_{D}$ and $\bar{a}_{F}$, respectively. The value of $\bar{a}_{D}$ indicates the minimum value of the vibration amplitude as soon as the parametric resonance occurs, or, if the critical point $C$ is exceeded. The value of $\bar{a}_{F}$ reflects the minimum value of the amplitude at the moment that the principal parametric resonance condition ceases. The variation of $\bar{a}_{D}$ and $\bar{a}_{F}$ with respect to $\varepsilon H_{2}$ is shown in Figure 8.

\subsection{Response-nonlinear damping curves}

In the previous analysis, a series of numerically simulated results have shown in detail how the nonlinear quadratic damping affects the response of the cantilever beam under parametric excitation. In this section, we continue to explore these effects from the numerical standpoint by defining the response-nonlinear damping curves. This curve is obtained from a specific vibration condition imposed to the structure under test by the excitation mechanism, and Figure 9 defines three distinct operating regions (marked as I, II, and III) that differ essentially in terms of the excitation frequency imposed to the system as well as resulting vibration 


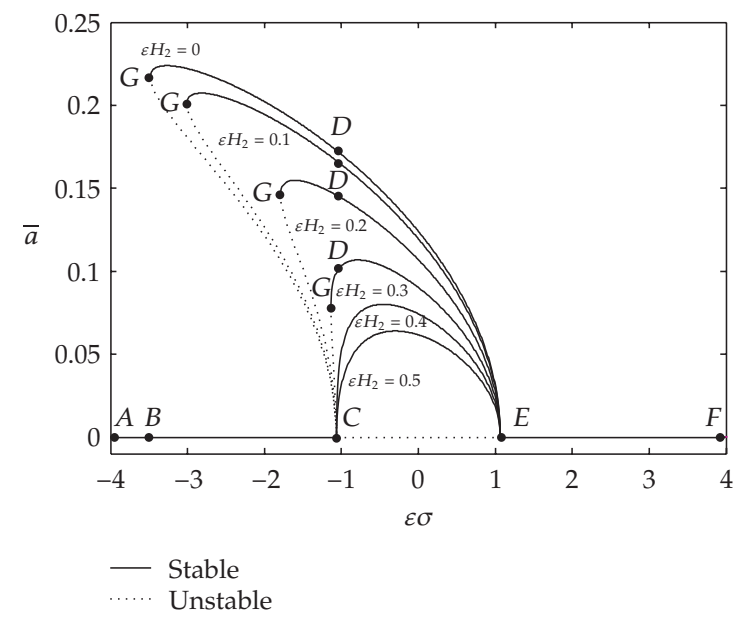

Figure 4: Typical response-frequency curve showing the effects of varying the quadratic dimensionless term $H_{2}$ and $\varepsilon H_{1}=0.0$ (nontrivial), $\varepsilon H_{1}=0.016$ (trivial), $\varepsilon H_{3}=2.1352, \varepsilon H_{4}=3.4674, \varepsilon H_{5}=62.7218$ [19].

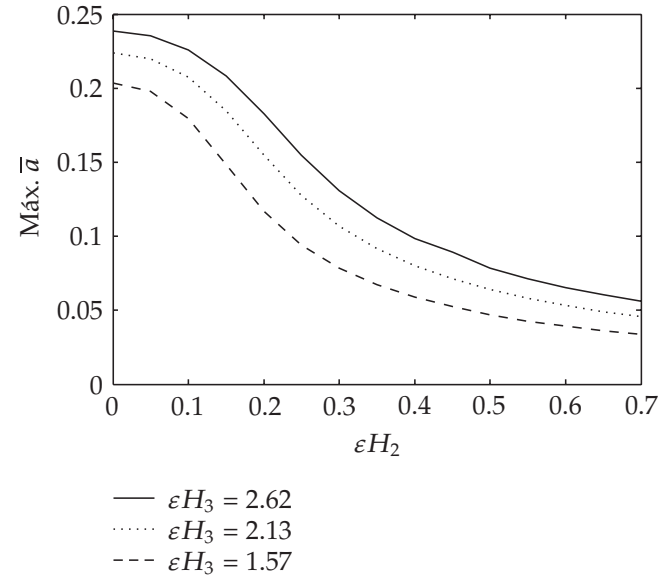

(a)

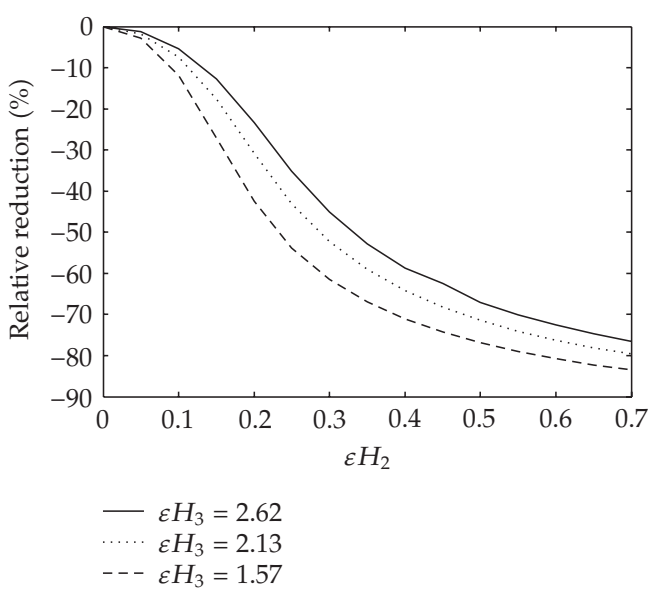

(b)

Figure 5: Influence of quadratic damping variation on the nontrivial response solution $\bar{a}$ : (a) variation of the maximum value of $\bar{a}$ with the nonlinear damping and (b) percent reduction of the maximum value of $\bar{a}$ for vacuum operation. Curves obtained for $(-) \varepsilon H_{1}=0.0, \varepsilon H_{3}=2.62, \varepsilon H_{4}=4.25, \varepsilon H_{5}=62.72 ;(\cdots)$ $\varepsilon H_{1}=0.0, \varepsilon H_{3}=2.13, \varepsilon H_{4}=3.46, \varepsilon H_{5}=62.72 ;(--) \varepsilon H_{1}=0.0, \varepsilon H_{3}=1.57, \varepsilon H_{4}=2.55, \varepsilon H_{5}=62.72$ [19].

amplitude. Two operation points $P 1$ and $P 2$ are chosen in regions I and II, respectively, with corresponding amplitudes given by $\bar{a}_{P 1}$ and $\bar{a}_{P 2}$. Figure 10 shows how variations on $\varepsilon H_{2}$ affect the amplitude $\bar{a}_{P 1}$. Two ramifications form this curve, one stable (solid line) and one unstable (dashed line). In case $\varepsilon H_{2}$ is decreased to the value $\varepsilon H_{2}=0$, the structure still remains vibrating but with an amplitude approximately $4 \%$ larger. Similarly, if $\varepsilon H_{2}$ increases beyond point $P 1$, the vibration amplitude decreases on the stable nontrivial ramification until point $C$ is reached. At this point, $\bar{a}_{P 1}$ is reduced by $19 \%$. If $\varepsilon H_{2}$ exceeds point $C$, the nontrivial stable solution 


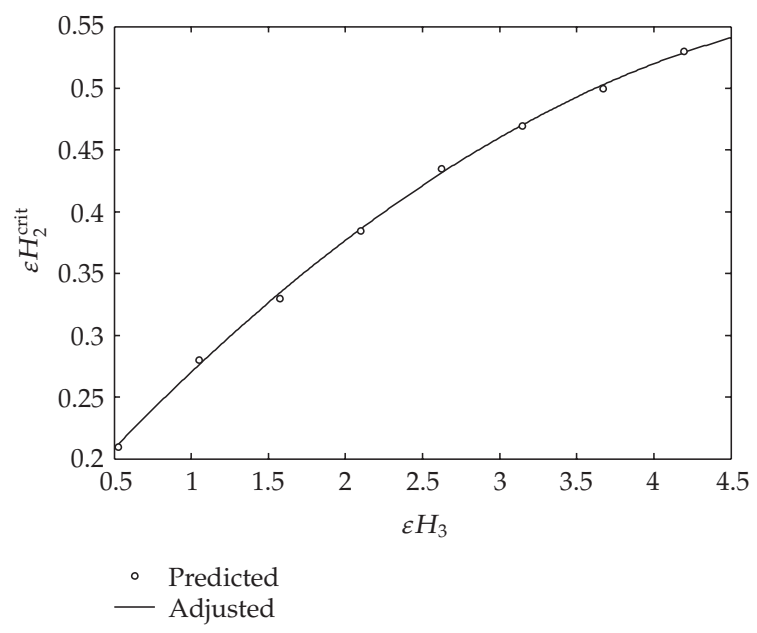

Figure 6: Critical values of $\varepsilon H_{2}$ as a function of $\varepsilon H_{3}$ [19].

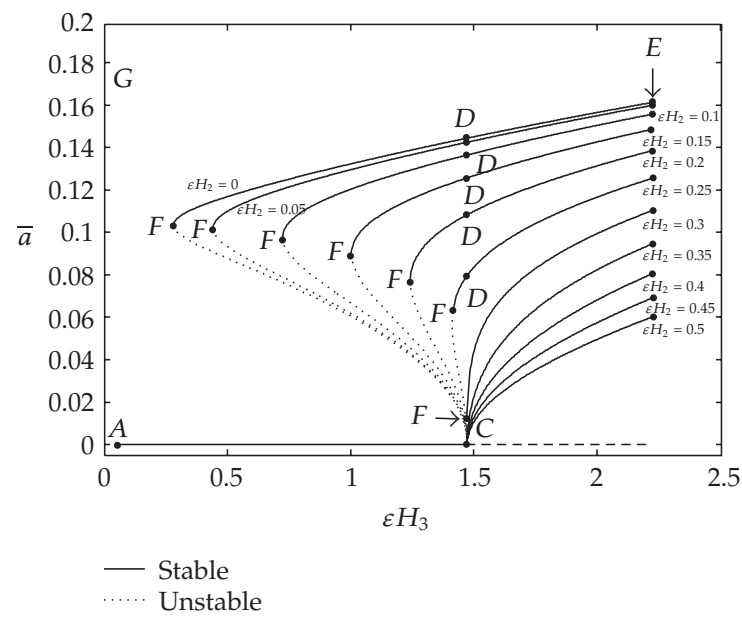

Figure 7: Typical response-amplitude curve demonstrating the effects of varying the quadratic nonlinear damping term $\left(\varepsilon H_{2}\right)$ for $\varepsilon \sigma=-0.73$. Also $\varepsilon H_{1}=0.0$ (nontrivial), $\varepsilon H_{1}=0.016$ (trivial), and $\varepsilon H_{5}=62.7218$ [19].

looses stability through a bifurcation and the vibration is extinct. When the structure vibrates according to point $P 2$, the magnitude of the response is given by $\bar{a}_{P 2}$. Figure 11 shows the effects on the system's response $\bar{a}_{P 2}$ produced by varying the values of $\varepsilon H_{2}$. In this case, a single stable ramification is observed, and independently on how $\varepsilon \mathrm{H}_{2}$ is varied the response continues being nontrivial and stable. On the other hand, there is a strong reduction in the value of $\bar{a}_{P 2}$. 


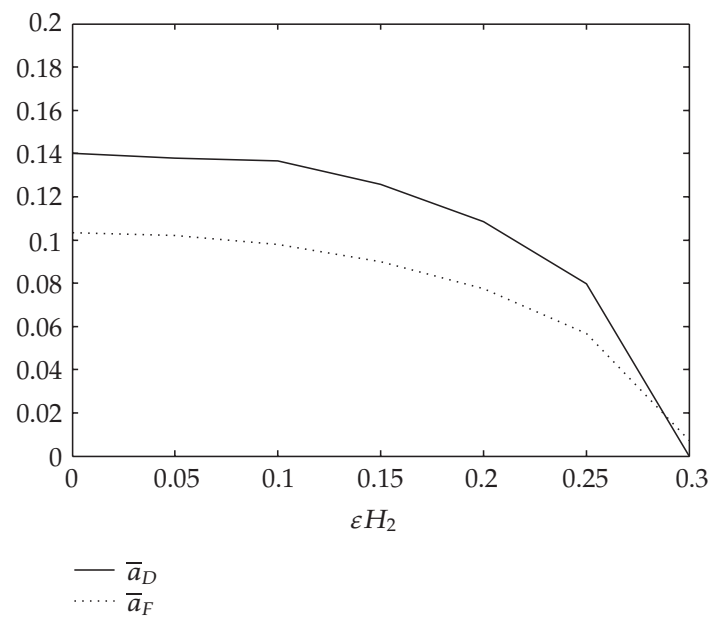

Figure 8: Typical plots showing the effects of the nonlinear quadratic damping on points $D$ and $F$. Results obtained with $\varepsilon \sigma=-0.73, \varepsilon H_{1}=0.0$ (nontrivial), $\varepsilon H_{1}=0.016$ (trivial), and $\varepsilon H_{5}=62.7218$ [19].

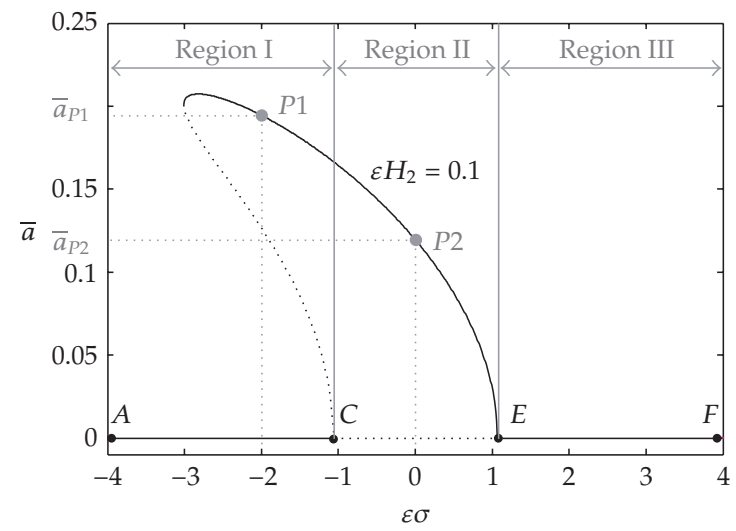

Figure 9: Typical response-frequency curve showing two specific operation points, designated as $P 1$ and $P 2$. Results obtained with $\varepsilon H_{1}=0.0$ (nontrivial), $\varepsilon H_{1}=0.016$ (trivial), $\varepsilon H_{2}=0.1, \varepsilon H_{3}=2.1352, \varepsilon H_{4}=$ 3.4674, $\varepsilon H_{5}=62.7218$ [19].

\section{Experimental analysis}

This section describes an experimental analysis that was performed on the structure shown in Figure 1. Initially some basic properties of the cantilever beam-mass system such as first bending damped natural frequency and modal damping ratio were obtained by standard modal testing procedures. In this case, the step relaxation method was employed to excite the system in order to get the driving point frequency response function (FRF) at the beam's end point [25]. The resulting values found for the first bending damped natural frequency and viscous modal damping ratio were $\omega_{d}=18.066 \mathrm{~Hz}$ and $\zeta_{m}=0.1272 \%$, respectively. These results were used to correlate the experimental results with the analytical prediction as well as in the planning of nonlinear tests. A detailed explanation of this procedure can be found in $[19,23]$. 


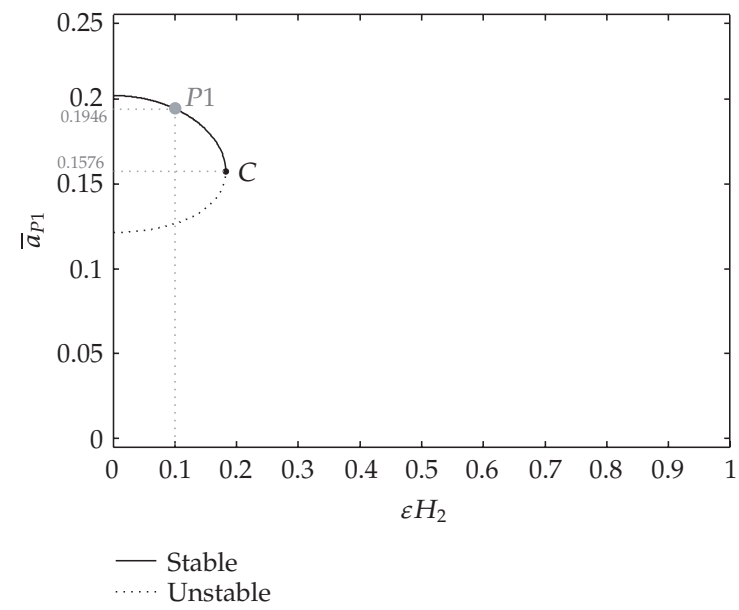

Figure 10: Typical response-quadratic damping curve showing the effects of variations of $\varepsilon \mathrm{H}_{2}$ on the magnitude of the response. Results obtained with $\varepsilon H_{1}=0.0$ (nontrivial), $\varepsilon H_{1}=0.016$ (trivial), $\varepsilon H_{3}=$ 2.1352, $\varepsilon H_{4}=3.4674, \varepsilon H_{5}=62.7218$, and $\varepsilon \sigma=-2[19]$.

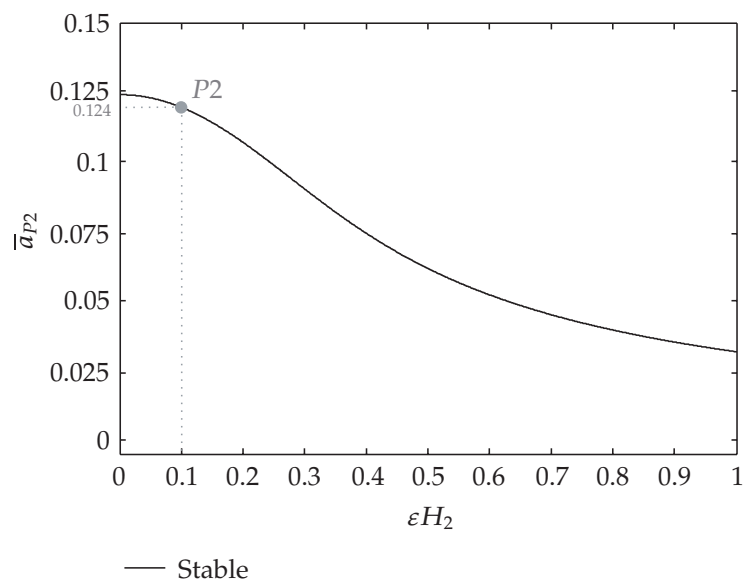

Figure 11: Typical response-quadratic damping curve showing the effects of variations of $\varepsilon \mathrm{H}_{2}$ on the magnitude of the response. Results obtained with $\varepsilon H_{1}=0.0$ (nontrivial), $\varepsilon H_{1}=0.016$ (trivial), $\varepsilon H_{3}=$ 2.1352, $\varepsilon H_{4}=3.4674, \varepsilon H_{5}=62.7218$, and $\varepsilon \sigma=0$ [19].

Once these basic linear characteristics were found, the system of Figure 1 was subjected to a base driven test according to the experimental setup shown in Figure 12. The beam carrying the lumped mass at one end is first attached at the opposite end to a steel block in order to properly simulate the fixed end boundary condition. This assembly is then mounted on the vibrating table of a B\&K type 4810 electrodynamic vibration exciter that will drive the system in the vertical direction. The excitation signal is provided by the HP Agilent E1432A data acquisition board that is controlled by the MTS I-Deas 10 modal testing software. The sinusoidal input signal was first sent to a B\&K power amplifier type 2707 


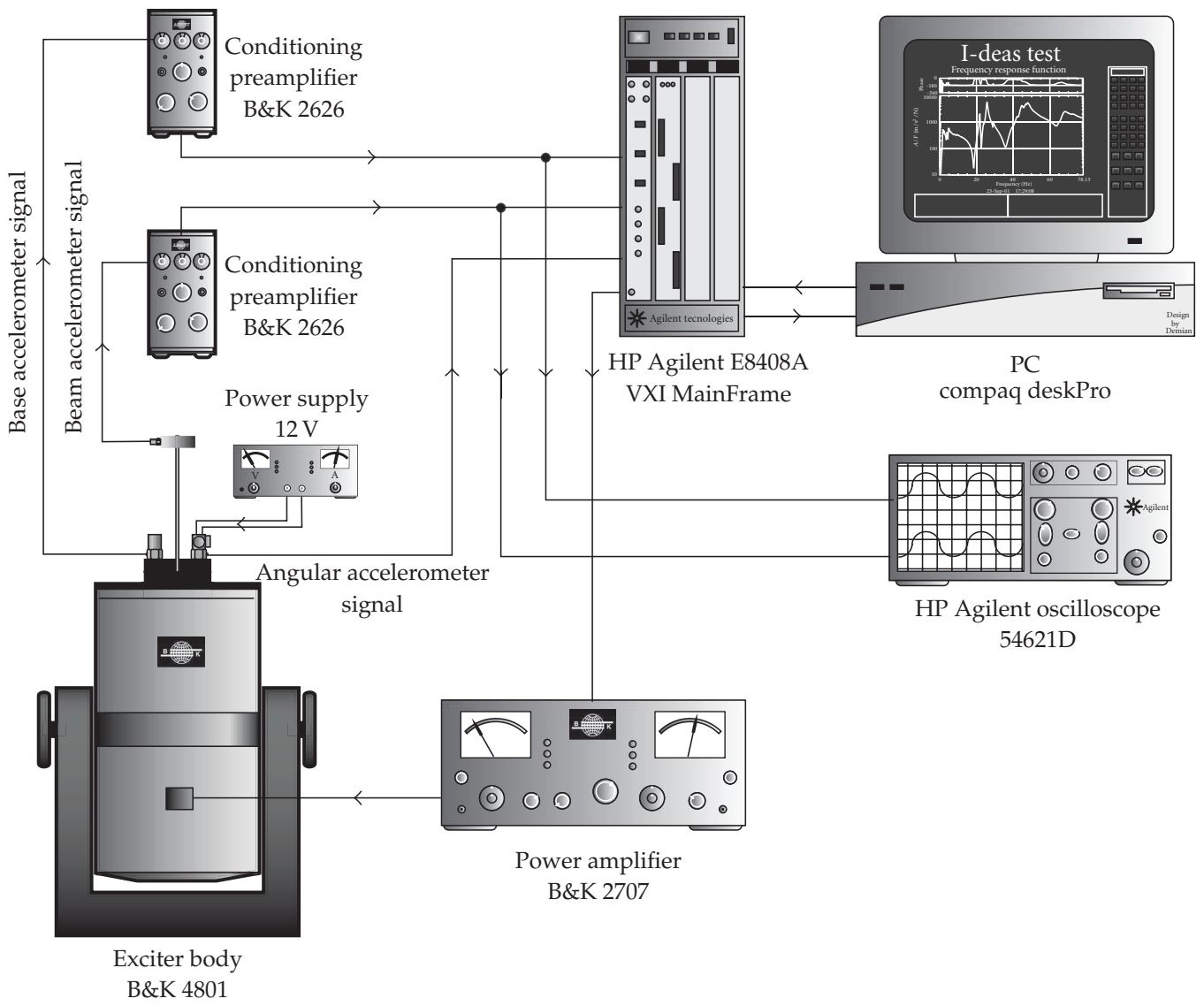

Figure 12: Experimental layout employed to obtain the experimental frequency-response and amplituderesponse curves [19-21].

and further sent to a B\&K type 4810 electrodynamic vibration exciter. The beam's transverse output signal was monitoring using an HP Agilent oscilloscope type 54621D. To minimize the rocking and translation, the shaker was clamped securely to the floor in the testing room. The sensing mechanism employed is three piezoelectric accelerometers, two for monitoring the base's motion and one for the beam-mass-system's motion. The base's linear translation motion was measured using an accelerometer B\&K model $4371(9.84 \mathrm{pC} / \mathrm{g})$, and possible rocking motion about $Y$ axis was measured using a Kistler angular accelerometer model 8836M01 $\left(34 \mu \mathrm{V} / \mathrm{rad} / \mathrm{s}^{2}\right)$. The beam-mass-system's translation motion was measured using an accelerometer B\&K model $4374(1.06 \mathrm{pC} / \mathrm{g})$ and mass of $0.64 \mathrm{~g}$.

In order to perform a coherent comparative analysis with the theoretical results, four experimental tests were conducted. In two of these tests, the amplitude of the base excitation acceleration was maintained constant at $39.24 \mathrm{~m} / \mathrm{s}^{2}$ and the sinusoidal excitation signal was slowly varied upward and downward in the frequency range of interest. The remaining tests were performed by keeping the excitation frequency constant at $36.132 \mathrm{~Hz}$, and then increasing the amplitude of the input excitation signal in the power amplifier. It should be noticed that the excitation frequency of $36.132 \mathrm{~Hz}$ corresponds to the so-called principal parametric excitation 


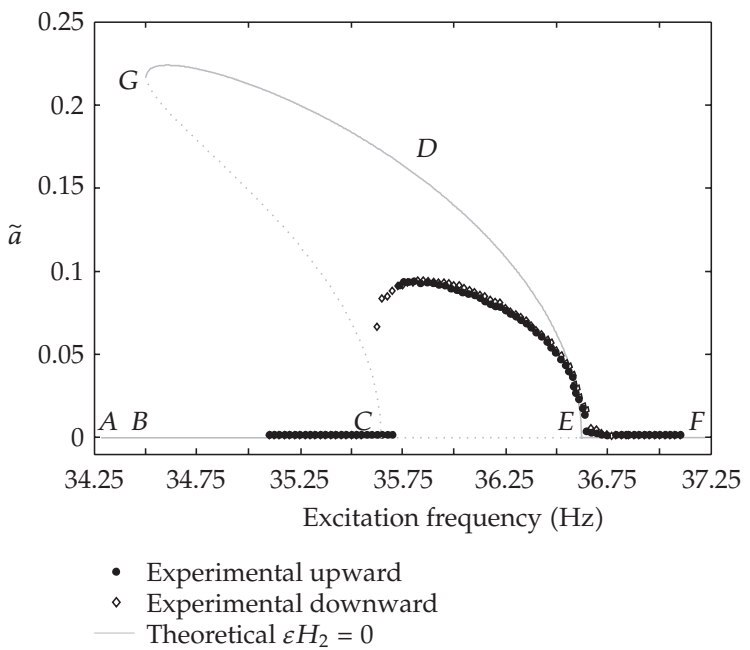

Figure 13: Experimental and theoretical response-frequency curves of the first flexural mode when $\varepsilon \mathrm{H}_{2}=$ 0.00 and for $\varepsilon H_{1}=0.0160$ (trivial), $\varepsilon H_{1}=0.0160$ (nontrivial), $\varepsilon H_{3}=2.1352 ; \varepsilon H_{4}=3.4674 ; \varepsilon H_{5}=62.7218$. Experimental results for parametric excitation frequency at $36.132 \mathrm{~Hz}$ and $39.24 \mathrm{~m} / \mathrm{s}^{2}$. (一) stable solution; $(\cdots)$ unstable solution [20].

frequency since it is approximately twice the system's first bending natural frequency thus satisfying the $2: 1$ relationship that is required to drive such a system into a principal parametric resonance condition [26].

The process of obtaining the experimental frequency-response curve consists of varying the excitation frequency while keeping the magnitude of the input base acceleration constant. The input base acceleration was maintained constant during the tests. A similar process was used to obtain the amplitude-response curves, however the frequency of excitation was kept constant and the amplitude was changed in small increments.

Figure 13 shows the experimental and theoretical frequency-response curves for the first bending mode. The theoretical curve was obtained in the absence of quadratic damping (i.e., $\varepsilon H_{2}=0.00$ ), while the experimental was obtained in atmospheric conditions.

The theoretical results in the upward direction show that by increasing the excitation frequency starting at point $A$, the trivial solution loses stability at point $C$, which corresponds to the critical value $\varepsilon \sigma=-\varepsilon H_{3} / 2$, through a subcritical pitchfork bifurcation and jumps up to point $D$. This point belongs to the nontrivial stable branch $G D E$. From point $D$, the steady-state amplitude of parametric response $\tilde{a}$ decreases as the excitation frequency is increased, until point $E$ is reached. From this point, the nontrivial solution loses stability through a supercritical pitchfork bifurcation and the trivial solution is reached again.

On the other hand, theoretical results in the downward direction show that decreasing the frequency of excitation form point $F$, the trivial solution looses stability at point $E$, corresponding to the critical value $\varepsilon \sigma=\varepsilon H_{3} / 2$, through a supercritical pitchfork bifurcation, and the nontrivial stable branch GDE is reached. From the point $E$, the response amplitude $\tilde{a}$ increases as the frequency is decreased. The solution loses stability though a turning point at point $G$ and the response amplitude jumps down to point $B$ where only the trivial solution exists thereafter. 


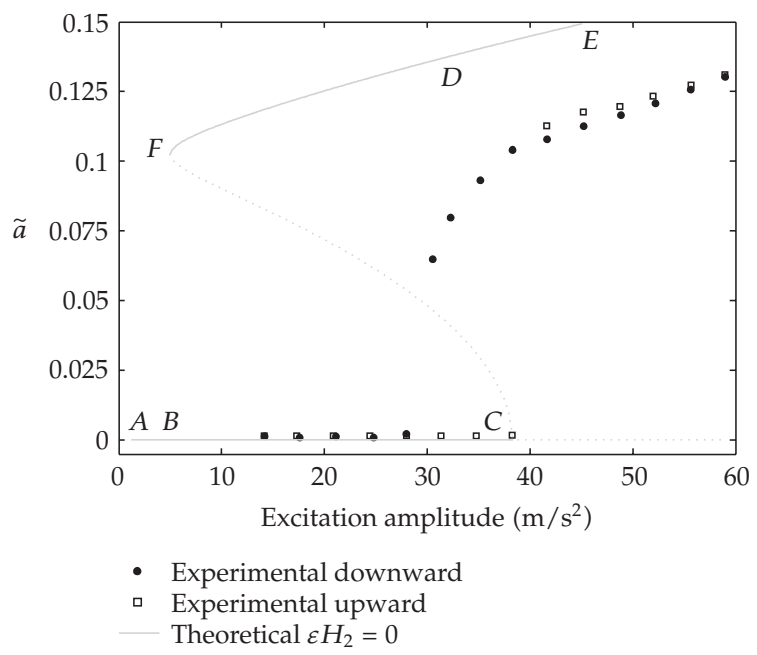

Figure 14: Experimental and theoretical amplitude-response curves of the first flexural mode when $\varepsilon \mathrm{H}_{2}=$ 0.00 and for $\varepsilon H_{1}=0.0160$ (trivial), $\varepsilon H_{1}=0.0160$ (nontrivial), $\varepsilon H_{3}=2.2236 ; \varepsilon H_{4}=3.6109 ; \varepsilon H_{5}=62.7218$ and $\varepsilon \sigma=-0.725$. Experimental results for parametric excitation frequency at $36.132 \mathrm{~Hz}$. (-) stable solution; $(\cdots)$ unstable solution [20].

When the theoretical behavior described above is confronted with the experimental results, it can be claimed that a good qualitative match between theory and experiment exists, mainly in the vicinity of the bifurcation frequencies. However, quantitatively there is an enormous difference between the amplitudes of theoretical and experimental results. The maximum theoretical value for the response amplitude is about $\tilde{a} \approx 0.225$, while the maximum experimental value is about $\tilde{a} \approx 0.1$, that is, about $125 \%$ smaller. This difference suggests the existence of important dissipative forces acting on the structure.

From this last result, it can be seen that by introducing the nonlinear quadratic damping a strong reduction of response amplitude has been achieved. Particularly, by using $\mathrm{eH}_{2}=$ 0.333 , an excellent agreement between the experimental and theoretical results was obtained. However, a slight discrepancy was observed in terms of the bifurcation points mainly in the upward sweep. Consequently, there is no exact agreement around point $C$. An analogous result was obtained for bifurcation point $E$.

Figure 14 shows the experimental and theoretical amplitude-response curves also for the first bending mode. Since an exact resonance condition $(\varepsilon \sigma=0)$ is difficult to be achieved, the theoretical curve was obtained for $\varepsilon \sigma<0$. In addition, the result shown was obtained in the absence of quadratic damping (i.e., $\varepsilon H_{2}=0.00$ ) while the experimental result was obtained in atmospheric conditions.

The theoretical results in the upward direction shows that by increasing the excitation amplitude from point $A$, the trivial solution loses stability at point $C$, corresponding to the critical value $\varepsilon H_{3}=\sqrt{4 \varepsilon \sigma^{2}+\varepsilon H_{1}^{2} \Theta^{2}}$, through a subcritical pitchfork bifurcation and jumps up to point $D$. This point belongs to the nontrivial stable branch FDE. A further increase in the excitation amplitude leads to higher response amplitudes tracing the branch $D E$.

On the other hand, the theoretical results in the downward direction shows that by decreasing the excitation amplitude from point $E$, the amplitude response continually 


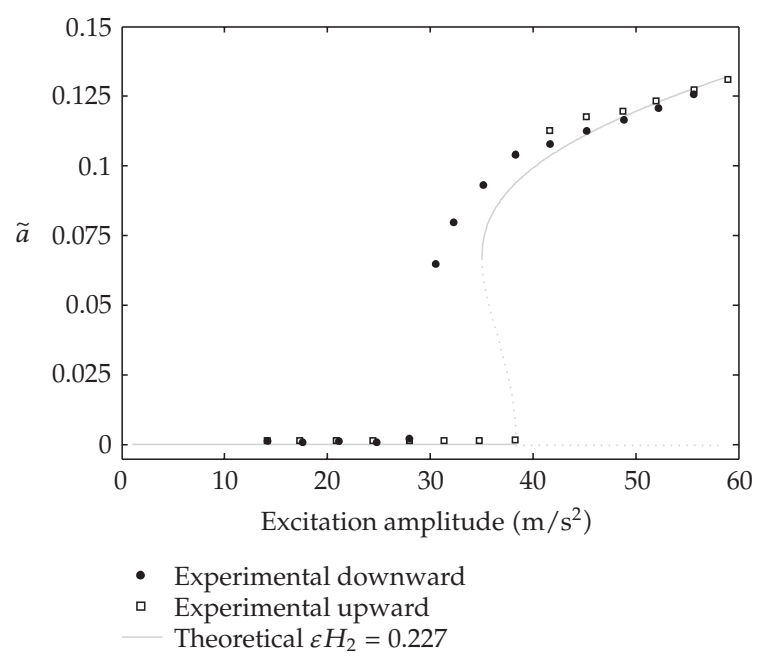

Figure 15: Experimental and theoretical amplitude-response curves of the first flexural mode when $\varepsilon \mathrm{H}_{2}=$ 0.227 and for $\varepsilon H_{1}=0.0160$ (trivial), $\varepsilon H_{1}=0.00$ (nontrivial), $\varepsilon H_{3}=2.2236, \varepsilon H_{4}=3.6109, \varepsilon H_{5}=62.7218$, and $\varepsilon \sigma=-0.725$. Experimental results for parametric excitation frequency at $36.132 \mathrm{~Hz}$. (-) stable solution; $(\cdots)$ unstable solution [20].

decreases until point $F$ is reached. Then, the nontrivial solution loses stability through a turning point bifurcation, leading to a jump down to point $B$ whereby only the trivial solution exists thereafter.

When the theoretical behavior described above is confronted with the experimental results, a very good qualitative as well as quantitative disagreement between them exists. These two characteristics also suggest the existence of important dissipative force acting on the structure. In order to prove this point, an additional theoretical amplitude-response curve was obtained, but now, including the nonlinear quadratic damping effects. The results are shown in Figure 15.

In this last figure, it can be claimed that by introducing the nonlinear quadratic damping a much better match between theory and experiment was obtained, specially when $\varepsilon \mathrm{H}_{2}=$ 0.227 is used. However, some discrepancies related to response amplitude, as well as with the bifurcation point $F$, were observed. A close view in region between $30 \mathrm{~m} / \mathrm{s}^{2}$ and $50 \mathrm{~m} / \mathrm{s}^{2}$ revealed $20 \%$ difference in the amplitude response. On the other hand, this discrepancy tends to decrease with the increase of the excitation amplitude. Also, there is a discrepancy related with the bifurcation point $F$. In the theoretical prediction, it occurs about $35 \mathrm{~m} / \mathrm{s}^{2}$, whereas in the experimental results it is shown at $30 \mathrm{~m} / \mathrm{s}^{2}$ showing a $17 \%$ difference.

\section{Concluding remarks}

This article addressed numerically and experimentally the effects of viscous fluid medium on the dynamic response of a cantilever beam carrying a lumped mass. Numerically simulated results showed the effects of variations induced in the nonlinear damping on the acceleration response of the test structure when it undergoes a principal parametric resonance condition. Experimental assessment on the effects of quadratic damping due to frictional turbulent force on the structure's dynamic response has been obtained. Generally speaking, good agreement 
between experimental and numerically simulated results was achieved in terms of frequency and amplitude response curves. It was observed that the quadratic damping due to frictional turbulent force plays an important role in the response of parametrically excited cantilever beam carrying a lumped mass. The inclusion of the quadratic damping significantly improves the theoretical predictions, and it should be included in the mathematical models when the problem involves the principal parametric response. Although the results shown in this paper were obtained for the first bending mode, similar conclusions may be obtained for higher natural frequencies.

\section{References}

[1] R. A. Ibrahim, "Structural dynamics with parameter uncertainties," Applied Mechanics Reviews, vol. 40, no. 3, pp. 309-328, 1987.

[2] S. Adhikari, "On the quantification of damping model uncertainty," Journal of Sound and Vibration, vol. 306, no. 1-2, pp. 153-171, 2007.

[3] J. Guo and X. Du, "Sensitivity analysis with mixture of epistemic and aleatory uncertainties," AIAA Journal, vol. 45, no. 9, pp. 2337-2349, 2007.

[4] J. R. Fonseca, M. I. Friswell, J. E. Mottershead, and A. W. Lees, "Uncertainty identification by the maximum likelihood method," Journal of Sound and Vibration, vol. 288, no. 3, pp. 587-599, 2005.

[5] C. Soize, "A comprehensive overview of a non-parametric probabilistic approach of model uncertainties for predictive models in structural dynamics," Journal of Sound and Vibration, vol. 288, no. 3, pp. 623-652, 2005.

[6] D. Moens and D. Vandepitte, "A fuzzy finite element procedure for the calculation of uncertain frequency-response functions of damped structures-part 1: procedure," Journal of Sound and Vibration, vol. 288, no. 3, pp. 431-462, 2005.

[7] H. De Gersem, D. Moens, W. Desmet, and D. Vandepitte, "A fuzzy finite element procedure for the calculation of uncertain frequency response functions of damped structures-part 2: numerical case studies," Journal of Sound and Vibration, vol. 288, no. 3, pp. 463-486, 2005.

[8] C. Chen, D. Duhamel, and C. Soize, "Probabilistic approach for model and data uncertainties and its experimental identification in structural dynamics: case of composite sandwich panels," Journal of Sound and Vibration, vol. 294, no. 1-2, pp. 64-81, 2006.

[9] A. Carcaterra, "Ensemble energy average and energy flow relationships for nonstationary vibrating systems," Journal of Sound and Vibration, vol. 288, no. 3, pp. 751-790, 2005.

[10] P. J. Shorter and R. S. Langley, "Vibro-acoustic analysis of complex systems," Journal of Sound and Vibration, vol. 288, no. 3, pp. 669-699, 2005.

[11] V. Cotoni, R. S. Langley, and M. R. F. Kidner, "Numerical and experimental validation of variance prediction in the statistical energy analysis of built-up systems," Journal of Sound and Vibration, vol. 288, no. 3, pp. 701-728, 2005.

[12] L. G. Crespo, D. P. Giesy, and S. P. Kenny, "Robustness analysis and robust design of uncertain systems," AIAA Journal, vol. 46, no. 2, pp. 388-396, 2008.

[13] C. L. Pettit, "Uncertainty quantification in aeroelasticity: recent results and research challenges," Journal of Aircraft, vol. 41, no. 5, pp. 1217-1229, 2004.

[14] J. M. Nichols, P. Marzocca, and A. Milanese, "On the use of the auto-bispectral density for detecting quadratic nonlinearity in structural systems," Journal of Sound and Vibration, vol. 312, no. 4-5, pp. 726$735,2008$.

[15] N. HaQuang, D. T. Mook, and R. H. Plaut, "A nonlinear analysis of the interactions between parametric and external excitations," Journal of Sound and Vibration, vol. 118, no. 3, pp. 425-439, 1987.

[16] N. HaQuang, D. T. Mook, and R. H. Plaut, "Non-linear structural vibrations under combined parametric and external excitations," Journal of Sound and Vibration, vol. 118, no. 2, pp. 291-306, 1987.

[17] A. H. Nayfeh and L. D. Zavodney, "Experimental observation of amplitude- and phase-modulated responses of two internally coupled oscillators to a harmonic excitation," Journal of Applied Mechanics, vol. 55, no. 3, pp. 706-710, 1988. 
[18] T. J. Anderson, A. H. Nayfeh, and B. Balachandran, "Experimental verification of the importance of the nonlinear curvature in the response of a cantilever beam," Journal of Vibration and Acoustics, vol. 118, no. 1, pp. 21-27, 1996.

[19] D. G. da Silva, Nonlinear vibrations in resonant beam type structures, Ph.D. thesis, Department of Mechanical Engeneering, University of São Paulo, Campus of São Carlos, Sao Carlos, Brazil, 2005.

[20] P S. Varoto and D. G. da Silva, "On the role of quadratic damping in the parametric response of a cantilever beam with tip mass: experimental investigation," in Proceedings of the 5th Euromech Nonlinear Dynamics Conference (ENOC '05), pp. 1-13, University of Technology, Eindhoven, The Netherlands, August 2005.

[21] P. S. Varoto and D. G. da Silva, "Nonlinear dynamic behavior of a flexible structure to combined external acoustic and parametric excitation," Shock and Vibration, vol. 13, no. 4-5, pp. 233-254, 2006.

[22] L. Meirovitch, Methods of Analytical Dynamics, McGraw-Hill, New York, NY, USA, 1970.

[23] D. G. da Silva and P. S. Varoto, "On the sufficiency of classical response models in predicting the dynamic behavior of flexible structures," in Proceeding of the 12th International Modal Analysis Conference (IMAC '04), pp. 1-21, SEM, Dearborn, Mich, USA, January 2004.

[24] M. Cartmell, Introduction to Linear, Parametric and Nonlinear Vibrations, Chapman and Hall, London, UK, 1990.

[25] K. G. McConnell, Vibration Testing: Theory and Pratice, John Wiley \& Sons, Chichester, UK, 1995.

[26] A. H. Nayfeh and D. T. Mook, Nonlinear Oscillations, Pure and Applied Mathematic, John Wiley \& Sons, New York, NY, USA, 1979. 


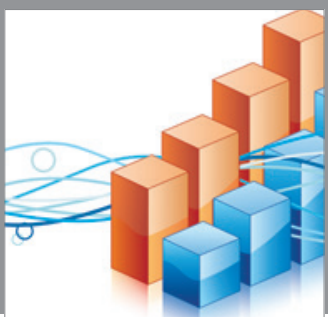

Advances in

Operations Research

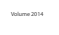

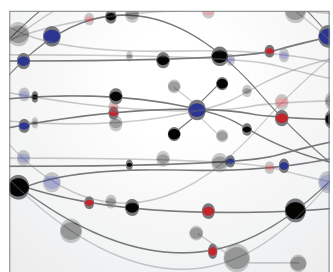

\section{The Scientific} World Journal
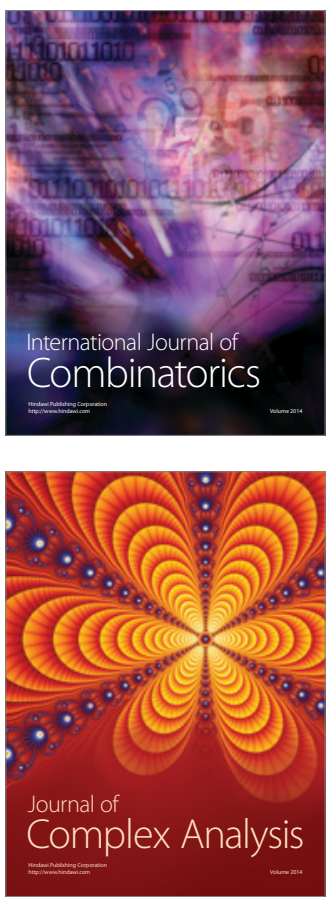

International Journal of

Mathematics and

Mathematical

Sciences
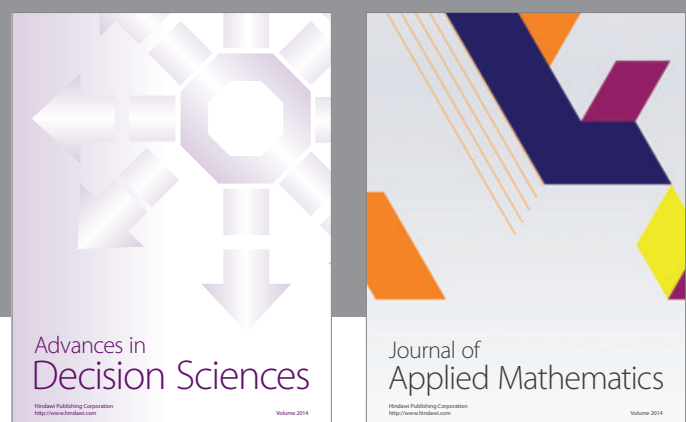

Journal of

Applied Mathematics
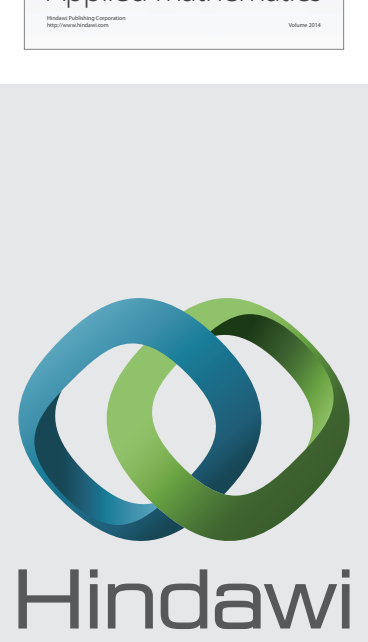

Submit your manuscripts at http://www.hindawi.com
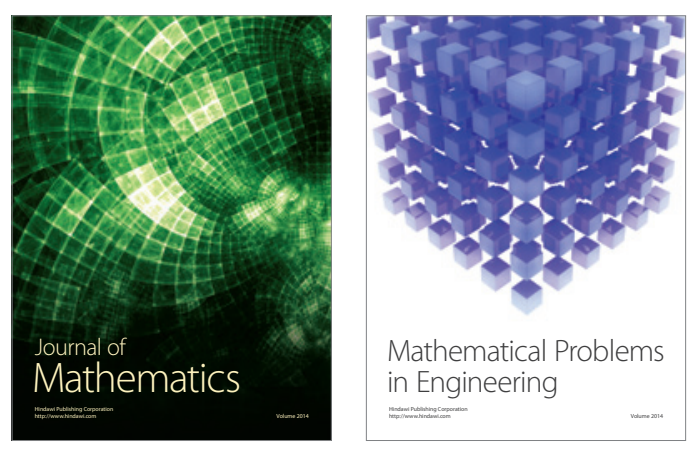

Mathematical Problems in Engineering
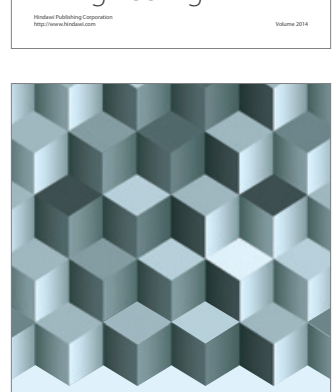

Journal of

Function Spaces
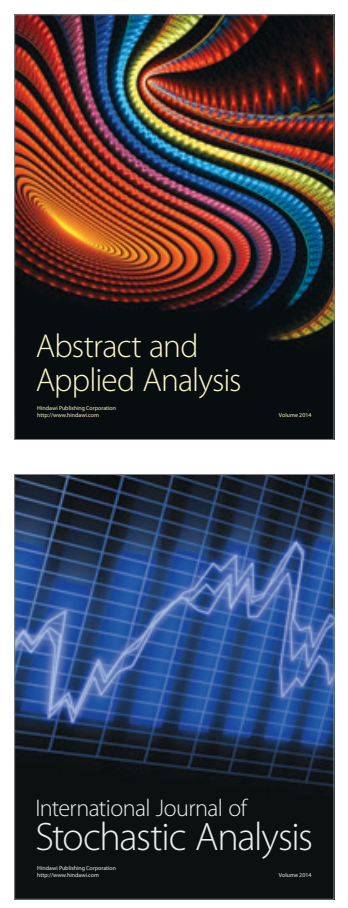

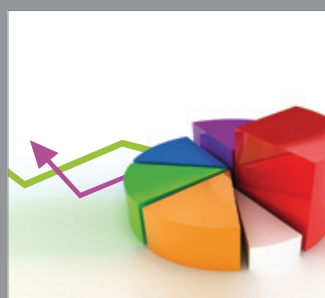

ournal of

Probability and Statistics

Promensencen
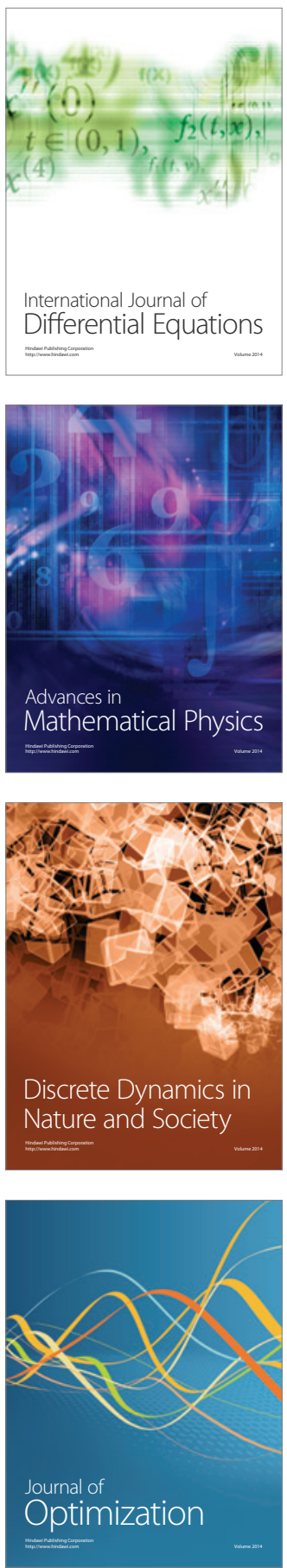